Fiaz Hussain • Ghulam Shabbir • Muhammad Ramzan

\title{
Classification of static cylindrically symmetric spacetimes in $f(R)$ theory of gravity by conformal motions with perfect fluid matter
}

Received: 19 February 2018 / Accepted: 15 July 2018 / Published online: 6 August 2018

(C) The Author(s) 2018

\begin{abstract}
Assuming the source of energy momentum tensor as perfect fluid, a classification of static cylindrically symmetric spacetimes in $f(R)$ theory of gravity by conformal vector fields (CVFs) is presented. For the classification purpose, we put different conditions on metric coefficients to obtain solutions in $f(R)$ theory of gravity. By means of some algebraic and direct integration techniques, it is shown that the dimension of CVFs for the considered spacetimes turns out to be 4,5 , or 15 .
\end{abstract}

الملخصص

بفرض أن موتر منبع طاقة الزخم مائع كامل، قدمنا تصنيفا للفضاء-الزمن الساكن والمتناظر أسطوانيا ضمن نظرية (R)

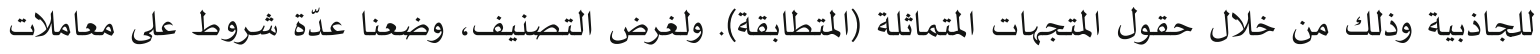

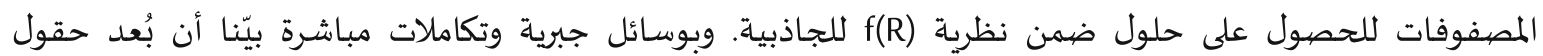

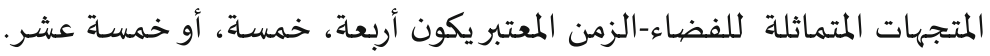

Mathematical Subject Classification $83 \mathrm{C} 05 \cdot 83 \mathrm{C} 15 \cdot 83 \mathrm{C} 20$

\section{Introduction}

No doubt, general theory of relativity is an elegant theory of gravitation to describe the structure of spacetime gravity and matter. However, there are some issues which arise from astrophysics and cosmology like the accelerated expansion of universe [14,27]. It is expected that this expansion is determined by an unknown form of energy called dark energy. An alternative for dark energy is assumed to be a cosmological constant [5]. To tackle such type of issues, a number of modifications in general relativity (GR) have been made. $f(R)$ theory is one of the modifications in GR introduced by Buchdahl in 1970, by making a suitable substitution of Ricci scalar R in Einstein Hilbert action [4]. Moreover, different $\mathrm{f}(\mathrm{R})$ models were proposed to justify the issues like late-time acceleration and early time inflation [12]. Use of these $f(R)$ models in Einstein field equations (EFEs) produces different exact solutions with constant and non-constant curvature. Solutions

F. Hussain · M. Ramzan ( $\varangle)$

Department of Mathematics, The Islamia University of Bahawalpur, Bahawalpur, Pakistan

E-mail: muhammad.ramzan@iub.edu.pk

G. Shabbir

Faculty of Engineering Sciences, GIK Institute of Engineering Sciences and Technology, Topi, Swabi, KPK, Pakistan

E-mail: shabbir@giki.edu.pk 
with constant curvature are in particular interest in $f(R)$ gravity. A detail of which could be found in [11]. However, finding exact solutions in $f(R)$ theory of gravity is very difficult as the equations of motion become highly non-linear. To overcome this problem, some symmetry restrictions like Killing symmetry, homothetic symmetry, and conformal symmetry, etc., are used which reduce the degrees of freedom of the problem and, hence, reduce the complexity of the problem. These symmetry restrictions also give conservation laws which describe the physical shape of the matter content as well as the geometrical features of the spacetime structure [7]. The class of spacetimes which is compatible with asymptotic flatness is the static spherically symmetric spacetimes containing matter. Cylindrically symmetric spacetimes are the next closest approach after spherical symmetry. The most general static cylindrical symmetric solutions were obtained by Levi-Civita [10] in 1917. After that, in the fluid case, a number of explicit cylindrical solutions have been obtained [26]. Moreover, by classifying spacetimes, according to some symmetry, restriction makes easier to find solutions of EFEs. A reasonable amount of work has been done on the classification of cylindrically symmetric spacetimes according to different symmetries in GR and teleparallel theory of gravitation [2,3,13,15-19]. Over the past few decades, finding exact solutions in $f(R)$ theory of gravity has remained a key subject for researchers using constant and non-constant curvature condition. A series of work in this era could be seen in $[1,21-24]$ and the references therein. Our purpose in this paper is to classify static cylindrically symmetric spacetimes in $f(R)$ theory of gravity by CVFs. CVFs have wide range of applications in astrophysics and cosmology. Looking towards the geometrical point of view, Maxwell's law of electromagnetic theory and light cone structures remains invariant under conformal transformations. Moreover, for massless particles along the null geodesics conformal symmetry produce constant of motion. A number of applications of CVFs in the theory of irreversible processes and many more could be seen in [8] and the references therein. Moreover, the role which CVFs play at the kinematic and dynamic level is documented in [9]. A conformal vector field $U$ is defined by [6]

$$
L_{U} g_{a b}=g_{a b, c} U^{c}+g_{b c} U_{, a}^{c}+g_{a c} U_{, b}^{c}=2 \psi g_{a b},
$$

where $\psi=\psi(t, r, \theta, z)$ is the smooth conformal function defined on the manifold $M, \quad L$ being the lie derivative along the vector field $U$, comma (,) denotes the partial derivative, and $g_{a b}$ is the metric tensor. If $\psi$ in the above Eq. (1.1) is a constant, then $U$ represents a homothetic vector field (proper homothetic if $\psi \neq 0$ ) and if it is zero, then $U$ becomes a Killing vector field. If the vector field $U$ is conformal but not homothetic, then it is called proper conformal vector field. It is clear from the definition that homothetic vector fields and Killing vector fields are special cases of CVFs.

\section{Field equations formulation in $f(R)$ gravity}

Consider a static cylindrically symmetric spacetimes in the usual coordinates $(t, r, \theta, z)$ (given by $\left(u^{0}, u^{1}, u^{2}, u^{3}\right)$ respectively) with the line element [26]

$$
\mathrm{d} s^{2}=-e^{\nu(r)} \mathrm{d} t^{2}+\mathrm{d} r^{2}+e^{\lambda(r)} \mathrm{d} \theta^{2}+e^{\mu(r)} \mathrm{d} z^{2}
$$

where $v=v(r), \lambda=\lambda(r)$, and $\mu=\mu(r)$ are non-zero functions of $r$ only. The above spacetimes (2.1) admit three linearly independent Killing vector fields which are [20]

$$
\frac{\partial}{\partial t}, \quad \frac{\partial}{\partial \theta}, \quad \frac{\partial}{\partial z} .
$$

The non-zero components of Ricci tensors for the spacetimes (2.1) are [25]

$$
\begin{aligned}
& R_{00}=\frac{1}{4} e^{v}\left[2 v^{\prime \prime}+v^{\prime 2}+v^{\prime} \lambda^{\prime}+v^{\prime} \mu^{\prime}\right] \\
& R_{11}=-\frac{1}{4}\left[2 v^{\prime \prime}+2 \lambda^{\prime \prime}+2 \mu^{\prime \prime}+v^{\prime 2}+\lambda^{\prime 2}+\mu^{\prime 2}\right], \\
& R_{22}=-\frac{1}{4} e^{\lambda}\left[2 \lambda^{\prime \prime}+\lambda^{\prime 2}+\lambda^{\prime} \mu^{\prime}+v^{\prime} \lambda^{\prime}\right], \\
& R_{33}=-\frac{1}{4} e^{\mu}\left[2 \mu^{\prime \prime}+\mu^{\prime 2}+\lambda^{\prime} v^{\prime}+v^{\prime} \mu^{\prime}\right]
\end{aligned}
$$


where overhead prime denotes the derivative with respect to $r$. Assuming the source of energy momentum tensor as perfect fluid i.e $T_{a b}=(\rho+p) s_{a} s_{b}+p g_{a b}$, where $\rho$ is the matter density, $p$ is the pressure, and $s_{a}$ is the fourvelocity vector which is defined as $s_{a}=-e^{\frac{v(r)}{2}} \delta_{a}^{0}$, we have $T_{00}=\rho e^{\nu}, \quad T_{11}=p, \quad T_{22}=p e^{\lambda}, \quad T_{33}=p e^{\mu}$. Field equations in $f(R)$ gravity are [21]

$$
H_{a b}=k T_{a b},
$$

where $H_{a b}=F(R) R_{a b}-\frac{1}{2} f(R) g_{a b}-\nabla_{a} \nabla_{b} F(R)+g_{a b} \square F(R), \quad f(R)$ is the function of Ricci scalar $R$, $F(R)=\frac{\mathrm{d}}{\mathrm{d} R} f(R), k$ is the coupling constant, $T_{a b}$ is the standard energy momentum tensor, and $\square=\nabla^{a} \nabla_{a}$ in which $\nabla_{a}$ is the covariant derivative operator. Using Eqs. (2.1) and (2.3) in Eq. (2.4) along with non-zero components of energy momentum tensor, we have

$$
\begin{aligned}
& F^{\prime \prime}-\frac{F^{\prime}}{2} v^{\prime}+\frac{F}{4}\left[2 \mu^{\prime \prime}+2 \lambda^{\prime \prime}+\lambda^{\prime 2}+\mu^{\prime 2}-v^{\prime} \lambda^{\prime}-v^{\prime} \mu^{\prime}\right]+k(\rho+p)=0 . \\
& \frac{F^{\prime}}{2}\left(\lambda^{\prime}-v^{\prime}\right)+\frac{F}{4}\left[2 \lambda^{\prime \prime}-2 v^{\prime \prime}+\lambda^{\prime 2}-v^{\prime 2}+\lambda^{\prime} \mu^{\prime}-v^{\prime} \mu^{\prime}\right]+k(\rho+p)=0 . \\
& \frac{F^{\prime}}{2}\left(\mu^{\prime}-v^{\prime}\right)+\frac{F}{4}\left[2 \mu^{\prime \prime}-2 v^{\prime \prime}+\mu^{\prime 2}-v^{\prime 2}+\lambda^{\prime} \mu^{\prime}-v^{\prime} \lambda^{\prime}\right]+k(\rho+p)=0 .
\end{aligned}
$$

To find the solutions of Eqs. (2.5-2.7), we classify spacetimes (2.1) by putting some restrictions on the metric coefficients along with the condition given in $F^{\prime}=0$ [22]. The classification has the following cases:

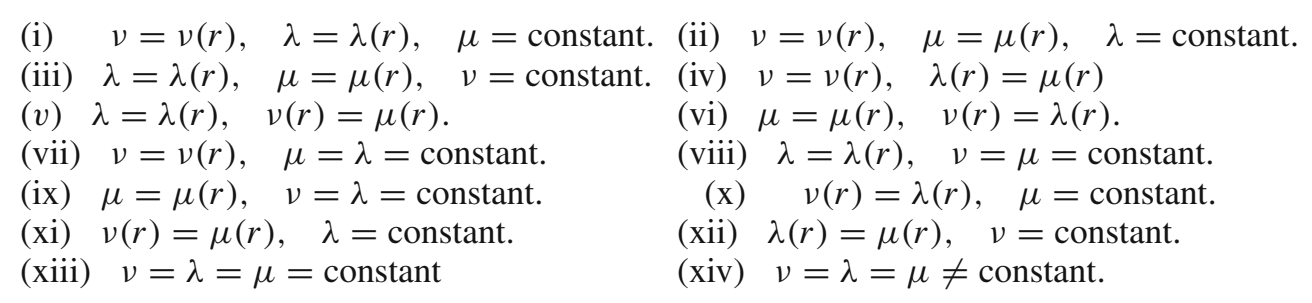

Solution of Eqs. (2.5-2.7) for the above cases is given in the upcoming portion of paper.

\section{Solution of field equations with CVFs}

In this section, we will find CVFs of the spacetime metrics obtained by finding the solution of Eqs. (2.5-2.7). Before finding the solutions of Eqs. (2.5-2.7), recall that a vector field $U$ is said to be conformal vector field if it satisfy Eq. (1.1), so using Eq. (2.1) in Eq. (1.1), one can obtain following ten first-order non-linear partial differential equations:

$$
\begin{aligned}
\dot{v} U^{1}+2 U_{, 0}^{0} & =2 \psi, \\
e^{v} U_{, 1}^{0}-U_{, 0}^{1} & =0, \\
e^{\nu} U_{, 2}^{0}-e^{\lambda} U_{, 0}^{2} & =0, \\
e^{\nu} U_{, 3}^{0}-e^{\mu} U_{, 0}^{3} & =0, \\
U_{, 1}^{1} & =\psi, \\
U_{, 2}^{1}+e^{\lambda} U_{, 1}^{2} & =0, \\
U_{, 3}^{1}+e^{\mu} U_{, 1}^{3} & =0, \\
\lambda^{\prime} U^{1}+2 U_{, 2}^{2} & =2 \psi, \\
e^{\lambda} U_{, 3}^{2}+e^{\mu} U_{, 2}^{3} & =0, \\
\mu^{\prime} U^{1}+2 U_{, 3}^{3} & =2 \psi .
\end{aligned}
$$

Solving Eqs. (3.3), (3.4), and (3.9) simultaneously, we have $U^{0}=\int F^{1}(t, r, \theta) \mathrm{d} \theta+F^{2}(t, r, z)$, where $F^{1}(t, r, \theta)$ and $F^{2}(t, r, z)$ are functions of integration. Now, using value of $U^{0}$ in Eqs. (3.2-3.4), we have 


$$
\begin{aligned}
U^{0}= & \int F^{1}(t, r, \theta) \mathrm{d} \theta+F^{2}(t, r, z) \\
U^{1}= & \int\left(e^{\nu} \int F_{r}^{1}(t, r, \theta) \mathrm{d} \theta\right) \mathrm{d} t+e^{\nu} \int F_{r}^{2}(t, r, z) \mathrm{d} t \\
& +F^{5}(r, \theta, z) \\
U^{2}= & e^{\nu-\lambda} \int F^{1}(t, r, \theta) \mathrm{d} t+F^{3}(r, \theta, z) \\
U^{3}= & e^{\nu-\mu} \int F_{z}^{2}(t, r, z) \mathrm{d} t+F^{4}(r, \theta, z)
\end{aligned}
$$

where $F^{3}(r, \theta, z), F^{4}(r, \theta, z)$, and $F^{5}(r, \theta, z)$ are functions of integration. Up to now, we have found the components of CVFs in terms of the unknown functions of integration and metric components. To find conformal vector field $U$, we have to solve Eqs. (3.1-3.10). It is important to mention that we are going to find conformal vector field $\mathrm{U}$ for the spacetimes metrics in $f(R)$ theory of gravity for the following cases.

Case (a): This case has the following three possibilities:

$$
\begin{gathered}
\text { (i) } \quad v=v(r), \quad \lambda=\lambda(r), \quad \mu=\text { constant. } \\
\text { (ii) } v=v(r), \quad \mu=\mu(r), \quad \lambda=\text { constant. } \\
\text { (iii) } \lambda=\lambda(r), \quad \mu=\mu(r), \quad v=\text { constant. }
\end{gathered}
$$

Using $\quad v=v(r), \quad \lambda=\lambda(r), \quad \mu=$ constant in Eqs. (2.5-2.7) after some simplifications gives

$$
2 \lambda^{\prime \prime}-2 v^{\prime \prime}-v^{\prime} \lambda^{\prime}-v^{\prime 2}+\lambda^{\prime 2}=0
$$

with two unknowns namely $\lambda$ and $\nu$. Now, to solve this equation, we assume solution of the form $v=k \lambda$, where $k \in \Re-\{0,1\}$. This assumption leads to $v=\ln \left(\frac{4}{c_{1} r+c_{2}}\right)^{4}$ and $\lambda=\ln \left(\frac{c_{1} r+c_{2}}{4}\right)^{4}$, and therefore, spacetime (2.1) after appropriate rescaling of $z$ takes the form:

$$
\mathrm{d} s^{2}=-\left(\frac{4}{c_{1} r+c_{2}}\right)^{4} \mathrm{~d} t^{2}+\mathrm{d} r^{2}+\left(\frac{c_{1} r+c_{2}}{4}\right)^{4} \mathrm{~d} \theta^{2}+\mathrm{d} z^{2}
$$

where $c_{1}, c_{2} \in \Re$. Now, solving Eqs. (3.1-3.10) for the spacetimes (3.13) and skipping lengthy and tedious calculations, one finds that $\psi=c_{3}$, which implies that CVFs become homothetic vector fields which are:

$$
\left.\begin{array}{rl}
U^{0} & =3 c_{3} t+c_{4} \\
U^{1} & =\left(\frac{c_{1} r+c_{2}}{c_{1}}\right) c_{3} \\
U^{2} & =-c_{3} \theta+c_{5} \\
U^{3} & =c_{3} z+c_{6}
\end{array}\right\},
$$

where $c_{3}, c_{4}, c_{5}, c_{6} \in \Re$. Generators of conformal algebra which are labeled by $X$ in this case are:

$$
X_{1}=3 t \frac{\partial}{\partial t}+\left(\frac{c_{1} r+c_{2}}{c_{1}}\right) \frac{\partial}{\partial r}-\theta \frac{\partial}{\partial \theta}+z \frac{\partial}{\partial z}, \quad X_{2}=\frac{\partial}{\partial t}, \quad X_{3}=\frac{\partial}{\partial \theta}, \quad X_{4}=\frac{\partial}{\partial z} .
$$

These generators form a closed-form conformal algebra whose non-zero commutation relations satisfy $\left[X_{1}, X_{2}\right]=-3 X_{2}, \quad\left[X_{2}, X_{1}\right]=3 X_{2}, \quad\left[X_{1}, X_{3}\right]=X_{3}, \quad\left[X_{3}, X_{1}\right]=-X_{3}\left[X_{1}, X_{4}\right]=-X_{4}$, $\left[X_{4}, X_{1}\right]=X_{4}$. Other possibilities (ii) and (iii) are exactly the same.

Case (b): The possibilities for this case are:

$\begin{aligned} & \text { (iv) } v=v(r), \quad \lambda(r)=\mu(r) . \\ & \text { (v) } \quad \lambda=\lambda(r), \quad v(r)=\mu(r) . \\ & \text { (vi) } \quad \mu=\mu(r), \quad v(r)=\lambda(r) .\end{aligned}$ 
When $v=v(r)$ and $\lambda(r)=\mu(r)$, Eqs. (2.5-2.7) give

$$
2 \lambda^{\prime \prime}-v^{\prime} \lambda^{\prime}+2 v^{\prime \prime}+v^{\prime 2}=0
$$

with two unknowns namely $\lambda$ and $\nu$. To solve (3.15), we assume solution of the form $v=k \lambda$, where $k \epsilon$ $\Re-\{0,1\}$. For $k=2$, solution of Eq. (3.15) turns out to be $v=\ln \left(c_{1} r+c_{2}\right)^{6}$ and $\lambda=\ln \left(c_{1} r+c_{2}\right)^{3}$, and therefore, spacetime (2.1) takes the form

$$
\mathrm{d} s^{2}=-\left(c_{1} r+c_{2}\right)^{6} \mathrm{~d} t^{2}+\mathrm{d} r^{2}+\left(c_{1} r+c_{2}\right)^{3}\left[\mathrm{~d} \theta^{2}+\mathrm{d} z^{2}\right],
$$

where $c_{1}, c_{2} \in \Re$. If one proceeds further using Eq. (3.16) and Eqs. (3.1-3.10), and avoiding from the lengthy calculations, one finds that CVFs become homothetic vector fields which are:

$$
\left.\begin{array}{rl}
U^{0} & =c_{3} t+c_{4} \\
U^{1} & =-\frac{c_{3}}{2}\left(\frac{c_{1} t+c_{2}}{c_{1}}\right) \\
U^{2} & =\frac{c_{3}}{4} \theta-c_{5} z+c_{6} \\
U^{3} & =\frac{c_{3}}{4} z+c_{5} \theta+c_{7}
\end{array}\right\},
$$

where $c_{3}, c_{4}, c_{5}, c_{6}, c_{7} \in \Re$. Conformal factor in this case is non-zero constant, i.e., $\psi=-\frac{c_{3}}{2}$. Generators of conformal algebra are:

$$
X_{1}=t \frac{\partial}{\partial t}-\left(\frac{c_{1} r+c_{2}}{2 c_{1}}\right) \frac{\partial}{\partial r}+\frac{\theta}{4} \frac{\partial}{\partial \theta}+\frac{z}{4} \frac{\partial}{\partial z}, \quad X_{2}=\theta \frac{\partial}{\partial z}-z \frac{\partial}{\partial \theta}, \quad X_{3}=\frac{\partial}{\partial t}, \quad X_{4}=\frac{\partial}{\partial \theta}, \quad X_{5}=\frac{\partial}{\partial z} .
$$

These generators form a closed-form conformal algebra whose non-zero commutation relations satisfy $\left[X_{1}, X_{4}\right]=-\frac{1}{4} X_{4}, \quad\left[X_{4}, X_{1}\right]=\frac{1}{4} X_{4}, \quad\left[X_{1}, X_{5}\right]=-\frac{1}{4} X_{5}, \quad\left[X_{5}, X_{1}\right]=\frac{1}{4} X_{5},\left[X_{2}, X_{4}\right]=-X_{5}$, $\left[X_{4}, X_{2}\right]=X_{5}, \quad\left[X_{2}, X_{5}\right]=X_{4}, \quad\left[X_{5}, X_{2}\right]=-X_{4}, \quad\left[X_{1}, X_{3}\right]=-X_{3}, \quad\left[X_{3}, X_{1}\right]=X_{3}$. Other possibilities (v) and (vi) are exactly the same. Case (c): This case has the following possibilities:

(vii) $\quad v=v(r), \quad \mu=\lambda=$ constant.

(viii) $\lambda=\lambda(r), \quad v=\mu=$ constant.

(ix) $\mu=\mu(r), \quad v=\lambda=$ constant.

When $v=v(r), \quad \mu=\lambda=$ constant. Equations (2.5-2.7) imply that $2 v^{\prime \prime}+v^{\prime 2}=0$, and therefore, $v=$ $\ln \left(c_{1} r+c_{2}\right)^{2}$, where $c_{1}, \quad c_{2} \in \Re\left(c_{1} \neq 0\right)$, and hence, spacetimes (2.1) after suitable rescaling of $\theta$ and $z$ take the form:

$$
\mathrm{d} s^{2}=-\left(c_{1} r+c_{2}\right)^{2} \mathrm{~d} t^{2}+\mathrm{d} r^{2}+\mathrm{d} \theta^{2}+\mathrm{d} z^{2} .
$$

For simplicity, one can choose $c_{1}=1$ and $c_{2}=0$, so that the above spacetimes (3.18) being conformally flat admit 15 independent CVFs which are the following:

$$
\left.\begin{array}{rl}
U^{0}= & \frac{1}{r}\left[\left(\frac{r^{2}+\theta^{2}+z^{2}}{2}\right)\left(c_{3} e^{t}+c_{4} e^{-t}\right)+z\left(c_{5} e^{t}+c_{10} e^{-t}\right)+\theta\left(c_{7} e^{t}+c_{8} e^{-t}\right)-c_{9} e^{t}+c_{10} e^{-t}\right] \\
& +c_{11} \\
U^{1}= & -\left[\left(\frac{\theta^{2}+z^{2}-r^{2}}{2}\right)\left(c_{3} e^{t}-c_{4} e^{-t}\right)+z\left(c_{5} e^{t}-c_{10} e^{-t}\right)+\theta\left(c_{7} e^{t}-c_{8} e^{-t}\right)-c_{12} r z+c_{13} r \theta\right] \\
& +c_{9} e^{t}+c_{10} e^{-t}+c_{14} r \\
U^{2}= & \left(\frac{r^{2}-\theta^{2}+z^{2}}{2}\right) c_{13}+c_{12} \theta z+c_{15} z+c_{14} \theta+r \theta\left(c_{3} e^{t}-c_{4} e^{-t}\right)+r\left(c_{7} e^{t}-c_{8} e^{-t}\right)+c_{16} \\
U^{3}= & \left(\frac{z^{2}-r^{2}-\theta^{2}}{2}\right) c_{12}-c_{13} \theta z-c_{15} \theta+c_{14} z+r z\left(c_{3} e^{t}-c_{4} e^{-t}\right)+r\left(c_{5} e^{t}-c_{6} e^{-t}\right)+c_{17}
\end{array}\right\}
$$


where $c_{3}, c_{4}, c_{5}, c_{6}, c_{7}, c_{8}, c_{9}, c_{10}, c_{11}, c_{12}, c_{13}, c_{14}, c_{15}, c_{16}, c_{17} \in \Re$. Conformal factor in this case is $\psi=$ $r\left(c_{3} e^{t}-c_{4} e^{-t}\right)+c_{12} z-c_{13} \theta+c_{14}$. Generators of conformal algebra are:

$$
\begin{aligned}
X_{1} & =e^{t}\left(\frac{r^{2}+\theta^{2}+z^{2}}{2 r}\right) \frac{\partial}{\partial t}-e^{t}\left(\frac{\theta^{2}+z^{2}-r^{2}}{2}\right) \frac{\partial}{\partial r}+e^{t} \theta r \frac{\partial}{\partial \theta}+e^{t} z r \frac{\partial}{\partial z}, \\
X_{2} & =e^{-t}\left(\frac{\partial}{\partial r}+\frac{1}{r} \frac{\partial}{\partial t}\right), \\
X_{3} & =e^{-t}\left(\frac{r^{2}+\theta^{2}+z^{2}}{2 r}\right) \frac{\partial}{\partial t}+e^{-t}\left(\frac{\theta^{2}+z^{2}-r^{2}}{2}\right) \frac{\partial}{\partial r}-e^{-t} \theta r \frac{\partial}{\partial \theta}-e^{-t} z r \frac{\partial}{\partial z}, \\
X_{4} & =e^{t}\left(\frac{\partial}{\partial r}-\frac{1}{r} \frac{\partial}{\partial t}\right), \\
X_{5} & =\left(\frac{r^{2}-\theta^{2}+z^{2}}{2}\right) \frac{\partial}{\partial \theta}-\theta z \frac{\partial}{\partial z}-r \theta \frac{\partial}{\partial r}, \quad X_{6}=\left(\frac{z^{2}-r^{2}-\theta^{2}}{2}\right) \frac{\partial}{\partial z}+r z \frac{\partial}{\partial r}+\theta z \frac{\partial}{\partial \theta}, \\
X_{7} & =\frac{\partial}{\partial t}, \quad X_{8}=e^{t}\left(\frac{z}{r} \frac{\partial}{\partial t}-z \frac{\partial}{\partial r}+r \frac{\partial}{\partial z}\right), \quad X_{9}=e^{-t}\left(\frac{z}{r} \frac{\partial}{\partial t}+z \frac{\partial}{\partial r}-r \frac{\partial}{\partial z}\right), \\
X_{10} & =e^{t}\left(\frac{\theta}{r} \frac{\partial}{\partial t}-\theta \frac{\partial}{\partial r}+r \frac{\partial}{\partial \theta}\right), \quad X_{11}=e^{-t}\left(\frac{\theta}{r} \frac{\partial}{\partial t}+\theta \frac{\partial}{\partial r}-r \frac{\partial}{\partial \theta}\right), X_{12}=r \frac{\partial}{\partial r}+\theta \frac{\partial}{\partial \theta}+z \frac{\partial}{\partial z}, \\
X_{13} & =z \frac{\partial}{\partial y}-\theta \frac{\partial}{\partial z}, X_{14}=\frac{\partial}{\partial \theta}, \quad X_{15}=\frac{\partial}{\partial z} .
\end{aligned}
$$

Possibilities (viii) and (ix) are exactly the same forming 15 dimensional conformal algebra.

Case (d): This case has the following four possibilities:

(x) $\quad \nu(r)=\lambda(r), \quad \mu=$ constant.

(xii) $\lambda(r)=\mu(r), \quad v=$ constant. (xi) $v(r)=\mu(r), \quad \lambda=$ constant.

(xiii) $v=\lambda=\mu=$ constant.

When $v(r)=\lambda(r), \quad \mu=$ constant. Equations (2.5-2.7) imply that $v^{\prime 2}=0$, and therefore, $v=\alpha$, and hence, spacetimes (2.1) after suitable rescaling of ' $z$ ' take the form:

$$
\mathrm{d} s^{2}=\mathrm{d} r^{2}+\beta \mathrm{d} z^{2}+\alpha\left[-\mathrm{d} t^{2}+\mathrm{d} \theta^{2}\right]
$$

where $\alpha, \beta \in \Re^{+}$. Solving Eqs. (3.1-3.10) for the spacetimes (3.20) by means of direct integration techniques and avoiding from lengthy calculations, we obtain 15 independent CVFs which are:

$$
\left.\begin{array}{rl}
U^{0} & =\left(\frac{\alpha t^{2}+r^{2}+\alpha \theta^{2}+\beta z^{2}}{2 \alpha}\right) c_{1}+c_{3} t \theta+c_{2} t r-c_{4} t z+c_{5} t+\frac{1}{\alpha} c_{6} r+c_{7} \theta+c_{8} z+c_{9} \\
U^{1} & =\left(\frac{\alpha t^{2}+r^{2}-\alpha \theta^{2}-\beta z^{2}}{2}\right) c_{2}+c_{1} t r+c_{3} r \theta-c_{4} r z+c_{6} t+c_{5} r+c_{10} \theta+c_{11} z+c_{12} \\
U^{2} & =\left(\frac{\alpha t^{2}-r^{2}+\alpha \theta^{2}-\beta z^{2}}{2 \alpha}\right) c_{3}+c_{1} t \theta+c_{2} \theta r-c_{4} \theta z+c_{7} t-\frac{1}{\alpha} c_{10} r+c_{5} \theta+c_{13} z+c_{14} \\
U^{3} & =\left(\frac{r^{2}-\alpha t^{2}+\alpha \theta^{2}-\beta z^{2}}{2 \beta}\right) c_{4}+c_{1} t z+c_{2} r z+c_{3} \theta z+\frac{\alpha}{\beta} c_{8} t-\frac{1}{\beta} c_{11} r-\frac{\alpha}{\beta} c_{13} \theta+c_{5} z+c_{15}
\end{array}\right\}
$$

where $c_{1}, c_{2}, c_{3}, c_{4}, c_{5}, c_{6}, c_{7}, c_{8}, c_{9}, c_{10}, c_{11}, c_{12}, c_{13}, c_{14}, c_{15} \in \Re$. Conformal factor in this case is $\psi=$ $c_{1} t+c_{2} r+c_{3} \theta-c_{4} z+c_{5}$. Generators of conformal algebra in this case are: 


$$
\begin{aligned}
& X_{1}=\left(\frac{\alpha t^{2}+r^{2}+\alpha \theta^{2}+\beta z^{2}}{2 \alpha}\right) \frac{\partial}{\partial t}+r t \frac{\partial}{\partial r}+t \theta \frac{\partial}{\partial \theta}+t z \frac{\partial}{\partial z}, \quad X_{2}=t \frac{\partial}{\partial t}+r \frac{\partial}{\partial r}+\theta \frac{\partial}{\partial \theta}+z \frac{\partial}{\partial z}, \\
& X_{3}=\left(\frac{\alpha t^{2}+r^{2}-\alpha \theta^{2}-\beta z^{2}}{2}\right) \frac{\partial}{\partial r}+r t \frac{\partial}{\partial t}+r \theta \frac{\partial}{\partial \theta}+r z \frac{\partial}{\partial z}, \quad X_{4}=\frac{r}{\alpha} \frac{\partial}{\partial t}+t \frac{\partial}{\partial r}, \quad X_{5}=\frac{\partial}{\partial t}, \\
& X_{6}=\left(\frac{\alpha t^{2}-r^{2}+\alpha \theta^{2}-\beta z^{2}}{2 \alpha}\right) \frac{\partial}{\partial \theta}+r \theta \frac{\partial}{\partial r}+t \theta \frac{\partial}{\partial t}+\theta z \frac{\partial}{\partial z}, \quad X_{7}=t \frac{\partial}{\partial \theta}+\theta \frac{\partial}{\partial t}, \quad X_{8}=\frac{\partial}{\partial r}, \\
& X_{9}=\left(\frac{r^{2}-\alpha t^{2}+\alpha \theta^{2}-\beta z^{2}}{2 \beta}\right) \frac{\partial}{\partial z}-r z \frac{\partial}{\partial r}-t z \frac{\partial}{\partial z}-t z \frac{\partial}{\partial t}, \quad X_{10}=z \frac{\partial}{\partial t}+\frac{\alpha t}{\beta} \frac{\partial}{\partial z}, \quad X_{11}=\frac{\partial}{\partial \theta}, \\
& X_{12}=\theta \frac{\partial}{\partial r}-\frac{r}{\alpha} \frac{\partial}{\partial \theta}, \quad X_{13}=z \frac{\partial}{\partial r}-\frac{r}{\beta} \frac{\partial}{\partial z}, \quad X_{14}=z \frac{\partial}{\partial \theta}-\frac{\alpha \theta}{\beta} \frac{\partial}{\partial z}, \quad X_{12}=\frac{\partial}{\partial z} .
\end{aligned}
$$

By adopting the same procedure as in the case (a), one can find the conformal algebra using the above generators. Possibilities (xi-xiii) are precisely the same giving 15 independent conformal vector fields.

Case (e): In this case, $v=\lambda=\mu \neq$ constant. Again, Eqs. (2.5-2.7) imply that $v=d_{1} r$, and therefore, spacetimes (2.1) take the form:

$$
\mathrm{d} s^{2}=\mathrm{d} r^{2}+e^{d_{1} r}\left[-\mathrm{d} t^{2}+\mathrm{d} \theta^{2}+\mathrm{d} z^{2}\right],
$$

where $d_{1} \in \Re \quad\left(d_{1} \neq 0\right)$. It is important to mention that the above spacetimes are space-like version of FLRW for $k=0$, and, therefore, admit 15 independent conformal vector fields which are:

$$
\left.\begin{array}{l}
U^{0}=\left(\frac{\left(t^{2}+\theta^{2}+z^{2}\right)}{2}+\frac{2 e^{-d_{1} r}}{d_{1}^{2}}\right) c_{1}-\frac{2}{d_{1}}\left(c_{2} t+c_{3}\right) e^{\frac{-d_{1} r}{2}}+\left(c_{4} t+c_{5}\right) z+\left(-c_{6} t+c_{7}\right) \theta+c_{8} t+c_{9} \\
U^{1}=\left(\frac{\left(t^{2}-\theta^{2}-z^{2}\right) e^{\frac{d_{1} r}{2}}}{2}+\frac{2 e^{\frac{-d_{1} r}{2}}}{d_{1}^{2}}\right) c_{2}+\frac{2}{d_{1}}\left(c_{6} \theta-c_{4} z-c_{1} t-c_{8}\right)-e^{\frac{d_{1} r}{2}}\left(c_{10} z+c_{11} \theta-c_{3} t-c_{12}\right) \\
U^{2}=\left(\frac{\left(z^{2}-t^{2}-\theta^{2}\right)}{2}+\frac{2 e^{-d_{1} r}}{d_{1}^{2}}\right) c_{6}-\frac{2}{d_{1}}\left(c_{2} \theta+c_{11}\right) e^{\frac{-d_{1} r}{2}}+\left(c_{4} \theta+c_{13}\right) z+\left(c_{1} t+c_{8}\right) \theta+c_{7} t+c_{14} \\
U^{3}=\left(\frac{\left(t^{2}-\theta^{2}+z^{2}\right)}{2}-\frac{2 e^{-d_{1} r}}{d_{1}^{2}}\right) c_{4}-\frac{2}{d_{1}}\left(c_{2} z+c_{10}\right) e^{\frac{-d_{1} r}{2}}+\left(c_{1} t+c_{8}\right) z-\left(c_{6} z+c_{13}\right) \theta+c_{5} t+c_{15}
\end{array}\right\} .
$$

Conformal factor in this case is

$$
\psi=\left(\frac{-e^{\frac{-d_{1} r}{2}}}{d_{1}}+\frac{d_{1}\left(t^{2}-\theta^{2}-z^{2}\right) e^{\frac{d_{1} r}{2}}}{4}\right) c_{2}-\frac{d_{1}}{2} e^{\frac{d_{1} r}{2}}\left(c_{10} z+c_{11} \theta-c_{3} t-c_{12}\right),
$$

where $c_{1}, c_{2}, c_{3}, c_{4}, c_{5}, c_{6}, c_{7}, c_{8}, c_{9}, c_{10}, c_{11}, c_{12}, c_{13}, c_{14}, c_{15} \in \Re$. Generators of conformal algebra in this case are:

$$
\begin{aligned}
& X_{1}=\left(\frac{\left(t^{2}+\theta^{2}+z^{2}\right)}{2}+\frac{2 e^{-d_{1} r}}{d_{1}^{2}}\right) \frac{\partial}{\partial t}-\frac{2 t}{d_{1}} \frac{\partial}{\partial r}+t \theta \frac{\partial}{\partial \theta}+t z \frac{\partial}{\partial z}, \quad X_{2}=t \frac{\partial}{\partial t}-\frac{2}{d_{1}} \frac{\partial}{\partial r}+\theta \frac{\partial}{\partial \theta}+z \frac{\partial}{\partial z}, \\
& X_{3}=\left(\frac{\left(t^{2}-\theta^{2}-z^{2}\right) e^{\frac{d_{1} r}{2}}}{2}+\frac{2 e^{\frac{-d_{1} r}{2}}}{d_{1}^{2}}\right) \frac{\partial}{\partial r}-\frac{2 t e^{\frac{-d_{1} r}{2}}}{d_{1}} \frac{\partial}{\partial t}-\frac{2 \theta e^{\frac{-d_{1} r}{2}}}{d_{1}} \frac{\partial}{\partial \theta}-\frac{2 z e^{\frac{-d_{1} r}{2}}}{d_{1}} \frac{\partial}{\partial z}, \quad X_{4}=\frac{\partial}{\partial t}, \\
& X_{5}=\left(\frac{\left(t^{2}-\theta^{2}+z^{2}\right)}{2}-\frac{2 e^{-d_{1} r}}{d_{1}^{2}}\right) \frac{\partial}{\partial z}-\frac{2 z}{d_{1}} \frac{\partial}{\partial r}+t z \frac{\partial}{\partial t}+\theta z \frac{\partial}{\partial \theta}, \quad X_{6}=t \frac{\partial}{\partial \theta}+\theta \frac{\partial}{\partial t}, \quad X_{7}=\frac{\partial}{\partial \theta}, \\
& X_{8}=\left(\frac{\left(z^{2}-\theta^{2}-t^{2}\right)}{2}+\frac{2 e^{-d_{1} r}}{d_{1}^{2}}\right) \frac{\partial}{\partial \theta}+\frac{2 \theta}{d_{1}} \frac{\partial}{\partial r}-z \theta \frac{\partial}{\partial z}-t \theta \frac{\partial}{\partial t}, \quad X_{9}=z \frac{\partial}{\partial t}+t \frac{\partial}{\partial z}, \quad X_{10}=\frac{\partial}{\partial z},
\end{aligned}
$$




$$
\begin{aligned}
& X_{11}=-\theta e^{\frac{d_{1} r}{2}} \frac{\partial}{\partial r}+\frac{-2}{d_{1}} e^{\frac{-d_{1} r}{2}} \frac{\partial}{\partial \theta}, \quad X_{12}=-z e^{\frac{d_{1} r}{2}} \frac{\partial}{\partial r}+\frac{-2}{d_{1}} e^{\frac{-d_{1} r}{2}} \frac{\partial}{\partial z}, \quad X_{13}=z \frac{\partial}{\partial \theta}-\theta \frac{\partial}{\partial z}, \\
& X_{14}=t e^{\frac{d_{1} r}{2}} \frac{\partial}{\partial r}+\frac{-2}{d_{1}} e^{\frac{-d_{1} r}{2}} \frac{\partial}{\partial t}, \quad X_{15}=e^{\frac{-d_{1} r}{2}} \frac{\partial}{\partial r} .
\end{aligned}
$$

By adopting the same procedure as in the case (a), one can find the conformal algebra using the above generators.

\section{Results and discussion}

A classification of static cylindrically symmetric spacetimes in the context of $f(R)$ theory of gravity by their CVFs is presented. CVFs are obtained using direct integration techniques. This study reveals that the dimension of CVFs for the considered spacetimes turns out to be 4,5 , or 15 . The results are discussed as follows:

In the case (a), spacetime (2.1) admits four linearly independent CVFs out of which three are Killing vector fields and one is proper homothetic vector field; see Eq. (3.14). For the case (b), again, CVFs become homothetic vector fields in which four are Killing vector fields and one is proper homothetic vector field which are given in Eq. (3.17). In the case (c), spacetimes (2.1) become conformally flat and, therefore, admit 15 independent CVFs; see Eq. (3.19). In the case (d), spacetimes (2.1) are again conformally flat and, therefore, admit 15 independent CVFs see Eq. (3.21). In the case (e), spacetimes (2.1) become space-like version of FLRW model for $k=0$ and admit 15 linearly independent CVFs, and are given in Eq. (3.23).

Open Access This article is distributed under the terms of the Creative Commons Attribution 4.0 International License (http:// creativecommons.org/licenses/by/4.0/), which permits unrestricted use, distribution, and reproduction in any medium, provided you give appropriate credit to the original author(s) and the source, provide a link to the Creative Commons license, and indicate if changes were made.

\section{References}

1. Azadi, A.; Momeni, D.; Nouri-Zonoz, M.: Cylinderical symmetric solutions in $\mathrm{f}(\mathrm{R})$ gravity. Phys. Lett. B 670(3), 210-214 (2008)

2. Bokhari, A.H.; Kashif, A.R.; Qadir, A.: A complete classification of curvature collineations of cylindrically symmetric static metrics. Gen. Rel. Grav. 35(6), 1059-1076 (2003)

3. Bokhari, A.H.: On a cylindrically symmetric static metric admitting proper matter collineations. II Nuovo. Cimento B 118(7), 725-727 (2003)

4. Buchdahl, H.A.: Non-linear lagrangian and cosmological theory. Mon. Not. Roy. Astron. Soc 150(1), 1-8 (1970)

5. Carroll, S.M.: The cosmological constant. Living. Rev. Relat. 4(1), 1-50 (2001)

6. Hall, G.S.: Symmetries and Curvature Structure in General Relativity. World Scientific, Singapore (2004)

7. Khan, S.; Ahmad, F.; Ali, A.; Hussain, T.: A note on Killing symmetries of Lemaitre-Bondi metric. J. Appl. Environ. Biol. Sci. 4(7S), 403-405 (2014)

8. Khan, S.; Hussain, T.; Bokhari, A.H.; Khan, G.A.: Conformal Killing vectors of plane symmetric four dimensional lorentzian manifolds. Eur. Phys. J. C. 75(11), 523-531 (2015)

9. Maartens, R.; Mason, D.P.; Tsamparlis, M.: Kinametic and dynamic properties of conformal Killing vectors in anisotropic fluids. J. Math. Phys. 27(12), 2987-2994 (1986)

10. Fjallborg, Mikael: Static cylindrically symmetric space-times. Class. Quant. Gravity 24(9), 2253-2270 (2007)

11. Momeni, D.; Gholizade, H.: A note on constant curvature solutions in cylindrically symmetric metric f(R) gravity. Int. J. Mod. Phys. D 18(11), 1719-1731 (2009)

12. Nojiri, S.; Odintsov, S. D.: Problems Of Modern Theoretical Physics. A Volume in Honour of Prof. Buchninder (TSPU publishing, Tomsk) (2008), vol. 266. arXiv:0807.0685.

13. Qadir, A.; Ziad, M.: Classification of static cylindrically symmetric space-times. II Nuovo Cimento 110(3), 277-290 (1995)

14. Riess, A.G.; et al.: Observational evidence from supernovae for an accelerating universe and a cosmological constant. Astrophys. J. 116(3), 1009-1038 (1998)

15. Shabbir, G.; Bokhari, A.H.; Kashif, A.R.: Proper curvature collineations in cyllinderically symmetric space-times. II Nuovo Cimento B 118(9), 873-886 (2003)

16. Shabbir, G.; Khan, A.; Khan, S.: A note on classification of teleparallel conformal vector fields of cylindrically symmetric static space-times in telleparallel theory of gravitation. Int. J. Theor. Phys. 52(4), 1182-1187 (2013)

17. Shabbir, G.; Khan, S.: Classification of cylindrically symmetric static space-times according to their Killing vector fields in teleparallel theory of gravitation. Mod. Phys. Lett. A 25(7), 525-535 (2010)

18. Shabbir, G.; Khan, S.: Classification of teleparallel homothetic vector fields in cylindrically symmetric static space-times in teleparallel theory of gravitation. Commun. Theor. Phys. 54(4), 675-678 (2010)

19. Shabbir, G.; Ramzan, M.: Classification of cylindrically symmetric static space-times according to their proper homothetic vector fields. Appl. Sci. 9, 148-154 (2007) 
20. Shabbir, G.; Siddiqui, A.A.: Proper affine vector fields in cylindrically symmetric static space-times. II Nuovo Cimento B 120(9), 939-949 (2005)

21. Shamir, F.; Raza, Z.: Dust static cylindrically symmetric solutions in $f(R)$ gravity. Commun. Theor. Phys. 62(3), 348-352 (2014)

22. Shamir, F.: Exploring plane symmetric solutions in $\mathrm{f}(\mathrm{R})$ gravity. J. Exp. Theor. Phys. 122(2), 331-337 (2016)

23. Sharif, M.; Arif, S.: Static cylindrically symmetric interior solutions in $f(R)$ gravity. Mod. Phys. Lett. A 27(25), Article ID. 1250138, 1-12 (2012)

24. Sharif, M.; Arif, S.: Non-vacuum static cylindrically symmetric solutions and energy distribution in $f(R)$ gravity. Astrophys. Space. Sci. 342(1), 237-243 (2012)

25. Sharif, M.: Symmetries of energy momentum tensor of cylindrically symmetric static space-times. J. Math. Phys. 45(4), 1532-1560 (2004)

26. Stephani, H.; Kramer, D.; MacCallum, M.A.H.; Hoenselears, C.; Herlt, E.: Exact Solutions of Einsteins Field Equations, 2nd edn. Cambridge University Press, Cambridge (2003)

27. Tonry, J.L.; et al.: Cosmological results from high supernova. Astrophys. J. 594(1), 1-24 (2003)

Publisher's Note Springer Nature remains neutral with regard to jurisdictional claims in published maps and institutional affiliations. 